\title{
Indexing current advances with DOI - at the Infocommunications Journal
}

\author{
Pal Varga
}

$\mathrm{T}$ HE vast domain of Infocommunications reach from the physics of wireless and wired communication channels, through traversing the information - in a secure way - to its destination(s) to analyzing the characteristics of that transmission.

Since the area is huge, categorizing advances is hard. We operate with keywords - index terms -, text-mining of research papers, and creating clusters based on similar set of areas involved in these papers. The survey papers that keep appearing in our journal is useful in this sense as well: connecting and summarizing the current knowledge of a field - even if it has just emerged. In order to help indexing of our journal papers and the ones cited inside, we encourage our authors to reference the DOI - Document Object Identifier - of their cited articles, and we make sure these DOIs point to the source of the document, making it easier for the readers to reach it directly. This activity is animated by DOI commissioners such as the Hungarian Academy of Sciences, who helps us assigning DOIs through the original DOI provider, CrossRef.

The six papers of this issue includes an invited survey and five papers that arrived to the open call. Let us have a brief overview of these papers.

In their survey paper on cellular MIMO systems, Fodor, Pap and Telek discussed recent advances in the field of Channel State Information (CSI) acquisition and managing the inherent tradeoff between using time, frequency and power resources for CSI acquisition and transmitting data symbols. As they describe, managing this tradeoff has a large impact on the achievable spectral efficiency in cellular systems, in which the number of transmit and receive antennas grows large. They make the point that the joint allocation of frequency, time and power resources is subject to constraints that depend on the specific pilot pattern, described in the paper.

In their study, Çiftlikli et. al. considers the transceiver design for multi-user MIMO (MU-MIMO) communications, in which a single transmitter adopts beamforming to simultaneously transmit information at the first time-slot. beamforming to simultaneously transmit information at first During the second time-slot, receivers cooperate to share specific results of OSIC detection in each user. They propose the maximum-likelihood (ML) approach to estimate the received symbols, claiming that their solution does not increase the system complexity significantly.

In his paper, Roman Ipanov describes, sythesizes, and discusses the various characteristics of the polyphase ( $p$-phase, where $p$ is the prime integer number) radar signal. This signal has an area of zero side lobes in a vicinity of the central peak of autocorrelation function. He shows that this signal represents a train from $\mathrm{p}$ coherent phase-code-shift keyed pulses, which are coded by complementary sequences of the p-ary D-code.

Our distinguished author, Sergey M. Smolskiy teamed up with Dmitrii I. Popov in their recent article on non-recursive rejection filters (RF) in the transient mode. They achieved RF modernization by its structure adjustment according to results of clutter edge detection, which leads to its effectiveness increase in the transient mode sequentially from one pulse to another.

Alawadi, Zaher and Molnar introduce novel methods for predicting behavior of elephant flows in Data Center Networks. In their paper, they empirically designed, implemented, and analyzed a new performance evaluation model for flow scheduling and flow congestion control algorithms used in data center networks based on multiple stochastic workloads to predict the value at risk of the elephant flows loss rate.

In their paper, $\mathrm{Li}, \mathrm{Wu}$ and Wang propose a Deep Web data source classification method based on text feature extension and extraction. The experimental results not only show that their model has significant advantages over the previous methods, but also prove that the use of the Attention mechanism can improve the precision without a huge increase in the cost of training time.

Let us again stop for awhile and think of the seventy years that has passed since our HTE, the Scientific Association for Infocommunications, has born. It must be hard for most of us, since we have not been involved in research those times - so let us remember the ten years for our Journal. In both cases, this is a year for celebration: remembering some legendary achievements, and aiming for new challenges.

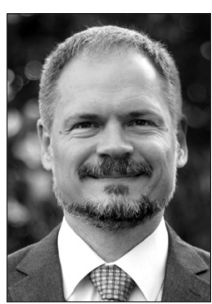

Pal Varga received the M.Sc. and Ph.D. degrees from the Budapest University of Technology and Economics, Hungary, in 1997 and 2011, respectively. $\mathrm{He}$ is currently an Associate Professor at the Budapest University of Technology and Economics. Besides, he is also the Director at AITIA International Inc. Earlier, he was working for Ericsson, Hungary, and Tecnomen, Ireland. His main research interests include communication systems, network performance measurements, root cause analysis, fault localisation, traffic classification, end-to-end QoS and SLA issues, as well as hardware acceleration. Recently he has been actively engaged with research related to Cyber-Physical Systems and Industrial Internet of Things. He has been involved in various industrial as well as European research and development projects in these topics. Besides being a member of HTE, he is a member of both the IEEE ComSoc (Communication Society) and IEEE IES (Industrial Electronics Society) communities, and the Editor-in-Chief of the Infocommunications Journal. 